\title{
DISTRIBUTION OF RANDOM ELEMENTS SUBJECTED TO A FLEXIBLE BOUNDARY CONDITION *
}

\author{
CHEN Han-dong (陈汉栋) ${ }^{1}, \quad$ QIAO Yu (乔宇 $)^{2}$ \\ (1. Department of Applied Physics, Stanford University, Stanford, CA 94305, USA; \\ 2. Department of Civil Engineering, University of Akron, Akron, OH 44325-3905, USA) \\ (Communicated by ZHOU Zhe-wei)
}

\begin{abstract}
The probability distribution function of $n$ random elements subjected to the flexible boundary condition is derived. The probability density is a descending curve and converges to a delta function as $n$ tends to infinity. The distribution of the minimum value is discussed in context of ordered statistics.
\end{abstract}

Key words: flexible boundary condition; constrained random elements; probability density; ordered statistics

Chinese Library Classification: O211.6

2000 Mathematics Subject Classification: 60G05

Digital Object Identifier(DOI): 10.1007/s 10483-006-0305-1

\section{Introduction}

The distribution of constrained random elements has been an active research area for decades $^{[1]}$. In this paper, we study the probability density, $p(x)$, of $n$ random elements $x_{i} \in$ $[0,1](i=1,2, \cdots, n ; n \geq 1)$ subjected to the flexible boundary condition

$$
\sum_{i=1}^{n} x_{i} \leq 1,
$$

which is of both primary scientific interest and immense technological importance. It is the basis of the discussion of the status of weakly bonded elementary particles ${ }^{[2]}$, the evolution of the collective microdefects in solid materials ${ }^{[3,4]}$, the optimization of traffic signal systems ${ }^{[5]}$, and so on. Although the distribution of the random elements with rigid boundary conditions, e.g., $\sum_{i=1}^{n} x_{i}=1$, has been well understood ${ }^{[6]}$, the studies on the flexible constraints are rare. This type of probability problems usually was solved either through Monte Carlo simulations or based on asymptotic distributions, which shed little light on the mechanisms or processes necessary for the eventual definitive control of the phenomena.

In addition to $p(x)$, the distribution of the minimum value of $x_{i}, p_{\mathrm{m}}(x)$, is also of important relevance in many cases. For example, the damage moment in a variety of crystalline materials is a single-value function of the probability of the presence of dislocation dipoles smaller than a critical size ${ }^{[7]}$. Currently, the only well established ordered statistics theory is for the sequences of random elements that are independent and identically distributed (i.i.d.) ${ }^{[8]}$. For random elements constrained by boundary conditions or internal processes, it can be prohibitively difficult to obtain the solution ${ }^{[9]}$.

In Section 1 below, $p(x)$ and $p_{\mathrm{m}}(x)$ will be investigated separately. We show that, through the conditional probability analysis, the analytical expressions can be derived and the theoretical results fit well with the data of Monte Carlo simulation.

\footnotetext{
* Received Mar.10, 2005; Revised Oct.18, 2005

Project supported by the National Science Foundation of USA (No.CMS-0503910)

Corresponding author QIAO Yu, Associate Professor, Doctor, E-mail: yqiao@uakron.edu
} 


\section{Main Results}

\subsection{Distribution of random elements}

Define

$$
y_{j}=y_{1}+\sum_{i=1}^{j-1} x_{i} \text { for } j \geq 2,
$$

with $j=2,3, \cdots, n+1$ and $y_{1}=0$. It can be seen that

$$
0=y_{1} \leq y_{2} \leq \cdots \leq y_{j} \leq y_{j+1} \leq \cdots \leq y_{n+1} \leq 1
$$

and $\left\{y_{j}\right\}$ distribute in $[0,1]$ randomly.

Without losing generality, let $y_{j+1}-y_{j}=\Delta(1 \leq j \leq n+1)$. For $0 \leq x \leq 1$,

$$
0=z_{1} \leq z_{2} \leq \cdots \leq z_{j} \leq z_{j+1} \leq \cdots \leq z_{n+1} \leq 1-\Delta,
$$

where

$$
z_{i}=\left\{\begin{array}{lll}
y_{i} & \text { for } & i \leq j \\
y_{i}-x & \text { for } & i>j
\end{array}\right.
$$

According to the conditional probability analysis, the probability of $\Delta \geq x$ can be stated as

$$
P(x)=\frac{\int_{0}^{1-x} d z_{n+1} \int_{0}^{z_{n+1}} d z_{n} \cdots \int_{0}^{z_{3}} d z_{2}}{\int_{0}^{1} d y_{n+1} \int_{0}^{y_{n+1}} d y_{n} \cdots \int_{0}^{y_{3}} d y_{2}}=(1-x)^{n} .
$$

Consequently, the probability density of the random elements $x_{i}$ is

$$
p(x)= \begin{cases}n(1-x)^{n-1}, & 0 \leq x \leq 1, \\ 0, & \text { elsewhere. }\end{cases}
$$

\subsection{Distribution of minimum value}

The minimum value of $x_{i}, x_{\mathrm{m}}$, should satisfy

$$
(n-1) x_{\mathrm{m}} \leq \sum_{i=1}^{n} x_{i}
$$

Thus, according to Eq.(1), we have

$$
x_{\mathrm{m}} \leq \frac{1}{n}
$$

Through Eq.(2), for a random variable $x \in\left[0, x_{\mathrm{m}}\right]$,

$$
x \leq y_{j+1}-y_{j}(j=1,2, \cdots, n),
$$

which can be rewritten as

$$
0=z_{1}^{\prime} \leq z_{2}^{\prime} \leq \cdots \leq z_{n+1}^{\prime} \leq 1-n x
$$

where

$$
z_{j}^{\prime}=y_{j}-(j-1) x .
$$

The probability of $x_{\mathrm{m}} \geq x(0 \leq x \leq 1 / n)$ is given by

$$
P_{\mathrm{m}}(x)=\frac{\int_{0}^{1-n x} d z_{n+1}^{\prime} \int_{0}^{z_{n+1}^{\prime}} d z_{n}^{\prime} \cdots \int_{0}^{z_{3}^{\prime}} d z_{2}^{\prime}}{\int_{0}^{1} d y_{n+1} \int_{0}^{y_{n}} d y_{n-1} \cdots \int_{0}^{y_{3}} d y_{2}}=(1-n x)^{n} .
$$

Consequently, the probability density of the minimum value of $x_{i}$ is

$$
p_{\mathrm{m}}(x)= \begin{cases}n^{2}(1-n x)^{n-1}, & 0 \leq x \leq 1 / n, \\ 0 & \text { elsewhere }\end{cases}
$$

Note that $p_{\mathrm{m}}(x)=n \cdot p(n x)$ in $0 \leq x \leq 1 / n$. 


\section{Discussion}

Equations (6) and (12) give the probability density of $x_{i}$ and the minimum value of $x_{i}$, respectively. Figure 1 shows the distribution of $x_{i}$ for different $n$. It can be seen that the theoretical results of Eq.(6) fit with the numerical data of the Monte Carlo simulation quite well. In the Monte Carlo simulation, the values of $x_{i}$ were produced by a random number generator. The process was repeated until 10000 valid sets of $x_{i}$ that satisfied Eq.(1) were generated. The statistical data indicate that the probability density always decreases with increasing $x$, with the peak at $x=0$. At $x=1, p(x)$ is always 0 . When $n=1$, the relationship between $p(x)$ and $x$ is linear. This linearity is lost when $n>1$, and the $p$-x curve converges to a delta function as $n$ tends to infinity. Through Eq.(6), the mean value of $x_{i}, E$, can be calculated as

$$
E=\frac{1}{n+1}
$$

and the standard deviation $\sigma$ is

$$
\sigma=\frac{1}{n+1} \sqrt{\frac{n}{n+2}} .
$$

Both $E$ and $\sigma$ decrease with increasing $n$, which is consistent with the $p$ - $x$ relation discussed above.

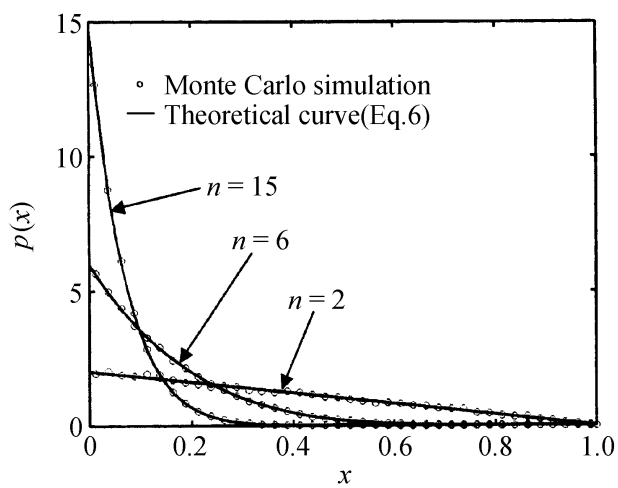

Fig.1 The probability density of the random variables $x_{i}$ subjected to the flexible boundary

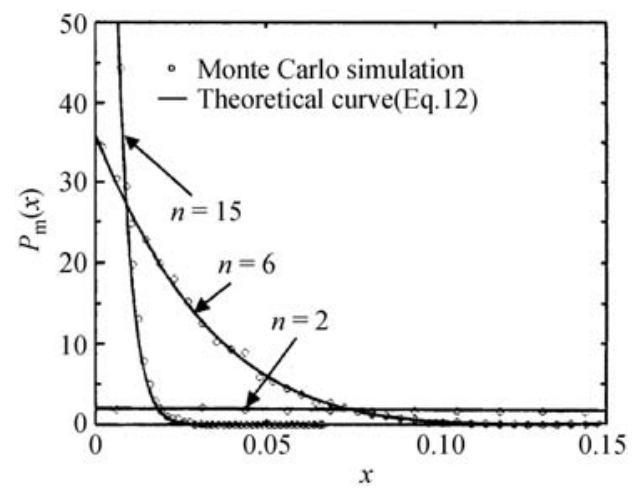

Fig.2 The probability density of the minimum value of $x_{i}$

Figure 2 shows the comparison of the theoretical results of Eq.(12) and the numerical data of the Monte Carlo simulation for the minimum value of $x_{i}$. The probability density of the minimum value decreases with increasing $x$, and for $n \geq 1$ and $x>E, p_{\mathrm{m}}(x)$ is always smaller than $p(x)$, as it should. Similar to $p(x)$, the $p_{\mathrm{m}}-x$ curve is linear when $n=1$, and converges to a delta function when $n$ tends to infinity. The mean value of the minimum $x_{i}, E_{\mathrm{m}}$, can be calculated through Eq.(12) as

$$
E_{\mathrm{m}}=\frac{1}{n(n+1)}=\frac{E}{n},
$$

and the standard deviation $\sigma_{\mathrm{m}}$ is

$$
\sigma_{\mathrm{m}}=\frac{1}{n+1} \sqrt{\frac{1}{n(n+2)}}=\frac{\sigma}{n} .
$$

Note that $E$ and $\sigma$ are larger than $E_{\mathrm{m}}$ and $\sigma_{\mathrm{m}}$ by a factor of $n$, respectively. 


\section{Conclusions}

In this article, the distribution of $n$ random elements $x_{i}$ subjected to the flexible boundary condition is discussed. The theoretical expressions of the probability density are obtained. The following conclusions are drawn:

(1) The probability density of $x_{i}$ is given by Eq.(6).

(2) The probability density of the minimum value of $x_{i}$ is given by Eq.(12).

(3) The mean values and the standard deviations are given by Eqs.(13)-(16).

(4) Both $p(x)$ and $p_{\mathrm{m}}(x)$ decrease as $x$ increases and converge to $\delta$-functions when $n \rightarrow \infty$.

\section{References}

[1] Kallenberg O. Foundations of Modern Probability[M]. Springer, New York, 1997.

[2] Lanyi C S, Liszi J. Monte-Carlo simulation of a low-density argon plasma[J]. Can J Appl Sepctrosc, 1993, 38(2): 37-41.

[3] Bai Y L, Xia M F, Ke F J, Li H L. Closed trans-scale statistical microdamage mechanics[J]. Acta Mech Sinica, 2002, 18(1): 1-17.

[4] Hong Y S, Qiao Y. An analysis on overall crack number density of short fatigue cracks[J]. Mech Mater, 1999, 31(8): 525-534.

[5] Park B B, Rouphail N M, Hummer J E. Probabilistic approach to implementing traffic signal warrants[J]. J Transp Eng ASCE, 2000, 126(4): 332-342.

[6] Darling D A. On a class of problems related to the random division of an interval[J]. Ann Math Stat, 1953, 24(2): 239-253.

[7] Qiao Y, Hong Y S. Collective behavior of short cracks and fatigue life prediction[J]. Adv Mech, 1997, 27(4): 489-503.

[8] Parzen E. Modern Probability Theory and Its Applications[M]. John Wiley \& Sons, New York, 1992.

[9] Chen D, Lu J C. The symptotics of maximum-likelihood estimates of parameters based on a data type where the failure and the censoring time are dependent[J]. Stat Prob Lett, 1998, 36(4): 379391. 